\title{
Stationary entanglement of atoms induced by classical field
}

Özgür Çakir, Alexander A. Klyachko, and Alexander S. Shumovsky

Citation: Appl. Phys. Lett. 86, 174103 (2005); doi: 10.1063/1.1915524

View online: http://dx.doi.org/10.1063/1.1915524

View Table of Contents: http://aip.scitation.org/toc/apl/86/17

Published by the American Institute of Physics

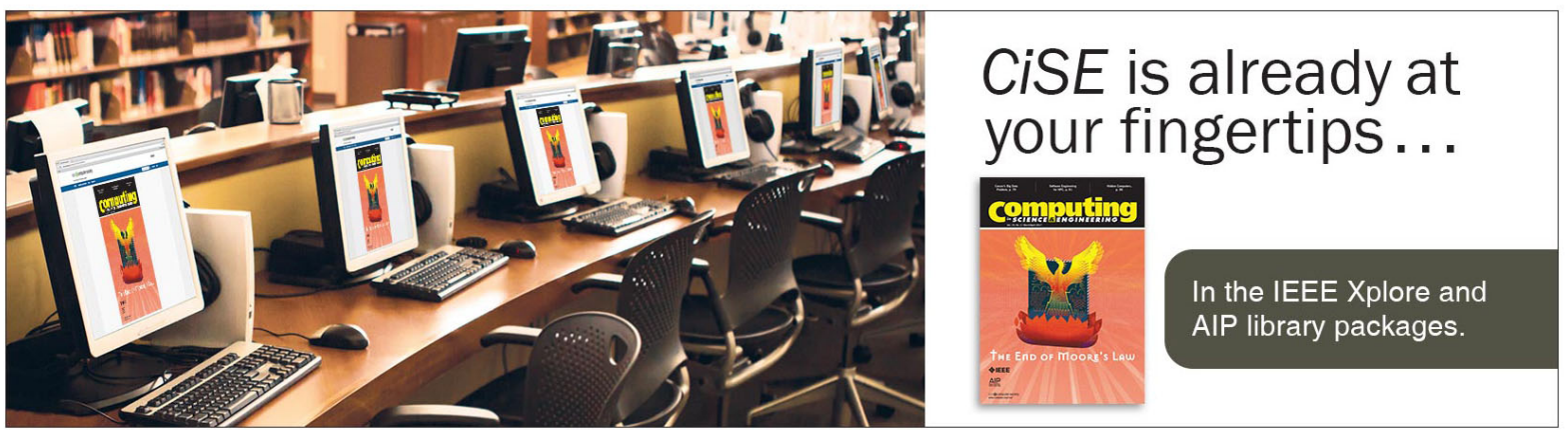




\title{
Stationary entanglement of atoms induced by classical field
}

\author{
Özgür Çakir, ${ }^{\text {a) }}$ Alexander A. Klyachko, and Alexander S. Shumovsky \\ Faculty of Science, Bilkent University, Bilkent Ankara, 06800 Turkey
}

(Received 28 July 2004; accepted 9 March 2005; published online 22 April 2005)

\begin{abstract}
We propose a scheme of stabilization of high-level entanglement through the use of classical driving field in atomic system with dipole-dipole interaction and losses caused by spontaneous radiation processes. () 2005 American Institute of Physics. [DOI: 10.1063/1.1915524]
\end{abstract}

Entanglement is known to be the main physical resource of quantum information processing and quantum computing (e.g., see Ref. 1 and references therein). At the same time, realization of quantum communication and computing protocols does not require just an entanglement but a robust one. This assumes a high amount of entanglement together with the long lifetime of entangled states.

It has been shown recently that entanglement in a system of three-level atoms in $\Lambda$ configuration can be stabilized by dissipation of the Stokes mode. ${ }^{2}$ The dissipation can also be used for stabilization of entanglement in a system of twolevel atoms with dipole-dipole interaction, located inside a lossy cavity. ${ }^{3}$ Another proposal on stabilization of entanglement of atoms with dipole-dipole interaction based on the use of squeezed vacuum field as a common electromagnetic bath for the atoms has been discussed in Ref. 4 .

We now note that the atomic systems represent a vivid model of qubits and can be successfully used as the main tool for modeling quantum devices and testing quantum information protocols. 5

The main aim of this letter is to discuss a way of stabilization of entanglement in a system of atoms with dipoledipole interaction and losses due to the spontaneous radiation processes, caused by the presence of a classical driving field.

The dipole-dipole interaction between the two identical two-level atoms located at $\mathbf{r}_{1}$ and $\mathbf{r}_{2}$, respectively, has the form $^{6}$

$$
H_{\mathrm{int}}=\hbar g\left(\sigma_{+}^{(1)} \sigma_{-}^{(2)}+H . c .\right),
$$

where $\sigma$ represents the atomic operators and

$$
g=\frac{|d|^{2}}{R^{3}}\left(1-3 \cos ^{2} \theta\right)=\frac{3 \Gamma_{0}}{4(k R)^{3}}\left(1-3 \cos ^{2} \theta\right),
$$

in the case of $1 \gg k_{0} R$, corresponding to the Lamb-Dicke limit. Here $\Gamma_{0}$ denotes the spontaneous-emission rate of photons with frequency $\omega=k c, R=\left|\mathbf{r}_{1}-\mathbf{r}_{2}\right|$ is the relative distance, and $\theta$ is the angle between the directions of dipoles $\mathbf{d}$ and interatomic axis $\mathbf{R}$.

The two eigenstates of the Hamiltonian (1)

$$
\left|\psi_{ \pm}\right\rangle=\frac{1}{\sqrt{2}}(|\uparrow \downarrow\rangle \pm|\downarrow \uparrow\rangle)
$$

with the eigenvalues $\epsilon_{ \pm}= \pm g$ correspond to the maximum entanglement of two dipoles (qubits). Thus, the system contains a potential ability to manifest entanglement. Undoubtedly, the losses caused by the spontaneous emission pro-

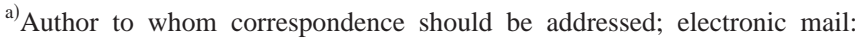
cakir@fen.bilkent.edu.tr
}

cesses would lead to irreversible evolution towards the unentangled state $|\downarrow \downarrow\rangle$ [since we are in the Lamb-Dicke limit, antisymmetric component in Eq. (3) will remain]. Therefore, to keep a certain amount of entanglement, it is necessary to connect the system with a "bath," compensating the losses of energy. We show here that the use of classical driving field as a bath stabilizes entanglement in the system on a good level suitable for further applications.

The interaction of two-level atoms with classical driving field can be described by the Hamiltonian

$$
H_{\mathrm{cdf}}=\sum_{i=1}^{2}\left(\frac{\Delta^{(i)}}{2} \sigma_{z}^{(i)}+\gamma e^{i \mathbf{k}_{0} \cdot \mathbf{r}_{i}} \sigma_{+}^{(i)}+\gamma e^{-i \mathbf{k}_{0} \cdot \mathbf{r}_{i}} \sigma_{-}^{(i)}\right),
$$

where $\gamma$ denotes the atom-field coupling constant. As usual, the classical driving field is considered as a monochromatic coherent field with high enough mean number of photons. Then, the irreversible evolution caused by the spontaneous emission of photons can be described by the master equation

$$
\dot{\rho}=-\frac{i}{\hbar}[H, \rho]+\frac{1}{2} \Gamma_{0} \sum_{i, j=1}^{2}\left(2 \sigma_{-}^{(i)} \rho \sigma_{+}^{(i)}-\sigma_{+}^{(i)} \sigma_{-}^{(j)} \rho-\rho \sigma_{-}^{(i)} \sigma_{-}^{(j)}\right),
$$

where $H=H_{\text {int }}+H_{\text {cdf }}$. The density matrix $\rho$ here is defined in the four-dimensional basis, consisting of the two maximally entangled states (3) and the two states $|\uparrow \uparrow\rangle$ and $|\downarrow \downarrow\rangle$. It can be easily seen that the $(4 \times 4)$ density matrix takes the block form

$$
\rho=\left(\begin{array}{cc}
\rho^{\prime} & 0 \\
0 & \rho^{\prime \prime}
\end{array}\right)
$$

where $\rho^{\prime}$ is the $(3 \times 3)$ matrix in the triple basis, consisting of the symmetric states of the two atoms and $\rho^{\prime \prime}$ is a $c$ number, the density matrix in the Hilbert space of singlet state.

Let us now note that the most natural initial state of the atomic system is $|\downarrow \downarrow\rangle$, when both atoms are in the ground state. It follows from Eq. (6) that the antisymmetric state $\left|\psi_{-}\right\rangle$ cannot be achieved in this case. Moreover, the system prepared in any symmetric initial state cannot evolve towards $\left|\psi_{-}\right\rangle$. At the same time, if the system is prepared initially in the maximally entangled state $\left|\psi_{-}\right\rangle$, it will state in this stay forever. Unfortunately, we do not know how to prepare this state.

Thus, beginning with the symmetric atomic state, we can discard $\left|\psi_{-}\right\rangle$and consider the evolution in the threedimensional sector of symmetric states specified by the reduced density matrix $\rho^{\prime}$ in Eq. (6). It should be stressed that in the absence of classical driving field the system evolves towards the unentangled atomic state $|\downarrow \downarrow\rangle$. 
Since we are interested in robust entanglement, we should consider the steady state solutions of the master Eq. (5) for the density matrix $\rho^{\prime}$ [Eq. (6)].

To solve the problem, we now assume the Lamb-Dicke limit of short interatomic distances ( $R$ is much less than the wavelength, so that $\left.k_{0} R \ll 1\right)$. This is just the case for trapped atoms $\left(R \sim 10^{-8} \div 10^{-9} \mathrm{~m}\right.$ and $k_{0} c$ is supposed to be the optical frequency). Under the further assumption that $\Delta=0$ and $g / \Gamma_{0}, \gamma / \Gamma_{0} \gg 1$, the steady state solution of Eq. (5) in the symmetric triplet sector takes the form

$$
\rho^{\prime}=\frac{1}{\tau^{2}+48}\left(\begin{array}{ccc}
16 & 0 & 4 i \tau \\
0 & 16 & 0 \\
-4 i \tau & 0 & 16+\tau^{2}
\end{array}\right),
$$

where $\tau=g \Gamma_{0} / \gamma^{2}$ is the dimensionless parameter.

The measure of entanglement in a two-qubit system is known to be the concurrence ${ }^{7}$

$$
C=\max \left(\lambda_{1}-\lambda_{2},-\lambda_{3}, 0\right),
$$

where $\lambda_{i}$ are the eigenvalues of the matrix $\sqrt{\rho^{\prime}} \bar{\rho}^{\prime} \sqrt{\rho^{\prime}}\left[\lambda_{1}\right.$ is the maximum eigenvalue and $\bar{\rho}$ denotes the complex conjugation of Eq. (7)]. It is now a straightforward matter to arrive at the relation

$$
C=\frac{8 \tau-16}{\tau^{2}+48}, \quad \tau \geqslant 2 .
$$

Thus, the maximum value of concurrence

$$
C_{\max }=(2 /(1+\sqrt{13}) \approx 0.43
$$

is achieved at

$$
\tau_{\max }=2(1+\sqrt{13}) \approx 9.21 .
$$

The corresponding amount of entanglement ${ }^{7}$ is

$$
\varepsilon_{\max } \approx 0.285 \text { ebit, }
$$

which seems to be high enough.

To estimate the possibility of experimental realization of the above described scheme, we now note that the dimensionless parameter $\tau$ can be rewritten as follows:

$$
\tau=\frac{3}{4 \pi \alpha}\left[\left(k_{0} R\right)^{3} Q n\right]^{-1},
$$

where $\alpha=1 / 137$ is the fine structure constant, $Q=k_{0} c T$ is the atomic "quality factor," $T$ is the lifetime of the excited atomic state, and $n$ is the mean number of photons interacting with atoms during the time $T$ (as usual, we assume here that $\gamma \sim \sqrt{n}$ ). Then, assuming that $R \sim 0.7 \times 10^{-3} \lambda_{0}$, where $\lambda_{0}$ denotes the wavelength of the classical driving field, we get $n \sim 10$. The above values of the key parameters seem to be accessible with the modern experimental technique. From Eq. (9) it is seen that the increase of the number of photons leads to decrease of the interatomic distance, which is necessary to provide the maximum amount of entanglement.

In the above estimations, we neglected the parameter $\Delta$, describing the detuning of the classical driving field from the atomic transition frequency. The numerical calculations show that the concurrence depends quite weakly on $\Delta$ at $\Delta / k_{0} c \ll 1$.

In the process of calculation of the result (8) we assumed that the polarization of classical driving field is parallel to the interatomic axis, so that the driving filed acts equivalently on both atoms. The alternative choice of the polarization orthogonal to $\mathbf{R}$ can lead to a strong change of the picture and deserves special consideration.

Summarizing, we have analyzed a way of creating robust entanglement in a system of two two-level atoms with dipole-dipole interaction. Physically, the entanglement is caused by the properties of the dipole-dipole interaction described by the Hamiltonian (1). Even if the system is prepared in the maximally entangled symmetric state $\left|\psi_{+}\right\rangle$[Eq. (3)], the irreversible evolution caused by spontaneous emission will lead to a rapid decay of entanglement. It is shown that the presence of the classical driving field leads to stabilization of entanglement. In this case, the level of entanglement is higher than that achieved through the use of a squeezed vacuum field instead of the classical driving field $(C \simeq 0.25$ in Ref. 4 ). It should be stressed that practically it is much easier to use the classical driving field than the squeezed vacuum field. Further it is possible to obtain an irreversible evolution, exactly of the form (5) for atoms driven by a coherent field in a damped cavity, as long as the atoms are strongly coupled with the cavity mode (see Ref. 8). In this case the atoms may, as well, be spatially well separated. The high-level entangled state in the system can be achieved starting from the very simple initial state of the system, e.g., $|\downarrow \downarrow\rangle$. The parameters, specifying the interatomic distances and driving field magnitude and corresponding to the maximum entanglement condition (8) seems to be realistic with the present level of experimental technique.

The authors wish to thank Professor A. Messina for useful comments.

${ }^{1}$ C. H. Bennett and P. W. Shor, IEEE Trans. Inf. Theory 44, 2724 (1998); M. A. Nielsen and I. L. Chuang, Quantum Computing and Quantum Information (Cambridge University Press, Cambridge, 2000).

${ }^{2}$ M. A. Can, A. A. Klyachko, and A. S. Shumovsky, Appl. Phys. Lett. 81, 5072 (2002); M. A. Can, Ö. Çakir, A. A. Klyachko, and A. S. Shumovsky, Phys. Rev. A 68, 022305 (2003); J. Opt. B: Quantum Semiclassical Opt. 6, S13 (2004).

${ }^{3}$ S. Nikolosi, A. Napoli, A. Messina, and F. Petruccione, E-print quant-ph/ 0402211 (to be published in Phys. Rev. A.)

${ }^{4}$ R. Tanaś and Z. Ficek, J. Opt. B: Quantum Semiclassical Opt. 6, S90 (2004); E-print quant-ph/0309195.

${ }^{5}$ G. Rempe, Ann. Phys. 9, 843 (2000); C. J. Miyatt, B. E. King, Q. A. Turchette, C. A. Sackett, D. Kielpinski, W. M. Itano, C. Monroe, and D. J. Wineland, Nature (London) 403, 269 (2000); J. M. Raymond, M. Brune, and S. Haroche, Rev. Mod. Phys. 73, 565 (2001); H. Walther, in Quantum Communication and Information Technologies, edited by A. S. Shumovsky and V. I. Rupasov (Kluwer, Dordrecht, 2003); E. S. Polzik, Nature (London) 428, 129 (2004).

${ }^{6}$ F. Seminara and C. Leonardi, Phys. Rev. A 42, 5695 (1990).

${ }^{7}$ S. Hill and W. K. Wooters, Phys. Rev. Lett. 78, 5022 (1997).

${ }^{8}$ J. Wang, H. M. Wiseman, and G. J. Milburn, E-print quant-ph/0409154 (2004). 